\title{
Nanocrown electrodes for robust and scalable intracellular recordings of cardiomyocytes for cardiotoxicity screening
}

\author{
Zeinab Jahed ${ }^{1,2^{*}}$, Yang Yang ${ }^{1 *}$, Ching-Ting Tsai ${ }^{1 *}$, Ethan P. Foster ${ }^{1}$, Allister F. McGuire ${ }^{1}$, Huaxiao \\ Yang $^{3,4}$, Aofei Liu ${ }^{1}$, Csaba Forro ${ }^{1}$, Zhenjie Yan ${ }^{5}$, Xin J. Ke ${ }^{5}$, Ming-Tao Zhao ${ }^{3,6,7}$, Wei Zhang ${ }^{1}$, Xiao \\ $\mathrm{Li}^{1}$, Thomas $\mathrm{Li}^{1}$, Annalisa Pawlosky ${ }^{8}$, Joseph C. $\mathrm{Wu}^{3,9,10}$, Bianxiao Cui ${ }^{1}$ \\ *co-first authors \\ ${ }^{1}$ Department of Chemistry, Stanford University, Stanford, CA 94305, USA \\ 2 Department of Nanoengineering, Jacobs school of Engineering, University of California, San Diego, CA 92039, USA \\ ${ }^{3}$ Stanford Cardiovascular Institute, Stanford University, Stanford, CA, USA. \\ ${ }^{4}$ Department of Biomedical Engineering, University of North Texas, Denton, TX, USA \\ ${ }^{5}$ Cyion Technologies, LLC., Pleasanton, CA 94566, USA \\ ${ }^{6}$ Center for Cardiovascular Research, Nationwide Children's Hospital, Columbus, OH, USA \\ 7 Department of Pediatrics, The Ohio State University College of Medicine, Columbus, OH, USA \\ ${ }^{8}$ Google Accelerated Sciences, Google LLC., Mountain View, 94043, CA USA \\ ${ }^{9}$ Department of Medicine, Stanford University, Stanford, CA, USA. \\ ${ }^{10}$ Department of Radiology, Stanford University, Stanford, CA, USA.
}

List of abbreviations

NEAs Nano-pillar Electrode Arrays

AP Action Potential

iAP Intracellular Action Potential

eAP Extracellular Action Potential 


\begin{abstract}
Drug-induced cardiotoxicity arises primarily when a compound alters the electrophysiological properties of cardiomyocytes. Features of intracellular action potentials (iAPs) are powerful biomarkers that predict proarrhythmic risks. However, the conventional patch clamp techniques for measuring iAPs are either laborious and low throughput or not suitable for measuring electrically connected cardiomyocytes. In the last decade, a number of vertical nanoelectrodes have been demonstrated to achieve parallel and minimally-invasive iAP recordings. Nanoelectrodes show great promise, but the large variability in success rate, signal strength, and the low throughput of device fabrication have hindered them from being broadly adopted for proarrhythmia drug assessment. In this work, we developed vertically-aligned and semi-hollow nanocrown electrodes that are mechanically robust and made through a scalable fabrication process. Nanocrown electrodes achieve $>99 \%$ success rates in obtaining intracellular access through electroporation, allowing reliable recordings of iAPs from human pluripotent stem-cellderived cardiomyocytes (hPSC-CMs). The accuracy of nanocrown electrode recordings is validated by simultaneous patch clamp recording from the same cell. Nanocrown electrodes enable prolonged iAP recording for continual monitoring of the same cells upon the sequential addition of four to five incremental drug doses. In this way, the dose-response data is selfreferencing, which avoids the cell-to-cell variations inherent to hPSC-CMs. We are hopeful that this technology development is a step towards establishing an iAP screening assay for preclinical evaluation of drug-induced arrhythmogenicity.
\end{abstract}

\title{
Introduction
}

The waveforms of intracellular action potentials (iAP) reflect the coordination of a multitude of ion channels, some of which are affected by drugs to collectively contribute toward proarrhythmic risks. Parameters including the action potential duration, diastolic interval, rate of depolarization, and the triangulation of repolarization of iAPs, have been shown to serve as predictors of proarrhythmia using human pluripotent stem-cell-derived cardiomyocytes (hPSC-CMs) (Hondeghem and Hoffmann 2003; Colatsky et al. 2016). The measurement of iAPs is mostly performed by patch clamp, which compares iAP waveforms before and after multi-dose drug treatment from the same cell, i.e. self-referencing. Manual patch clamp is reliable and accurate, but is limited by low throughput and high labor costs. For example, most manual patch clamp studies measure 3-7 hPSC-CMs per drug dose (Gibson et al. 2014; Liang et al. 2013; Crumb et al. 2016; Hyun et al. 2017). Several automated patch clamp techniques have been shown to increase the throughput (Scheel et al. 2014; Kramer et al. 2020; Dunlop et al. 2008), but these methods require the use of isolated cells in suspension, which disrupts electrically connected cardiomyocytes and may compromise the relevance of the measurement. Even with automated patches, studies usually measure $<10$ cells per drug dose (Scheel et al. 2014; Li et al. 2019; Stoelzle et al. 2011). Noninvasive and multiplexable microelectrodes can easily achieve parallel recording of extracellular action potentials (eAPs). However, eAPs appear as biphasic spikes and do not provide all crucial information for proarrhythmia assessment. Recent microelectrode-based 
local extracellular action potential method can capture many iAP features but it lacks one-to-one cell to electrode correspondence, is not self-referencing, and the accuracy of measured iAP features is yet to be fully validated by patch clamp (Hayes et al. 2019; Lopez et al. 2018; Edwards et al. 2018).

In the last decade, vertically-aligned and solid-state nanoelectrode arrays (NEAs) have emerged as promising tools with the potential of achieving parallelizable and minimally invasive iAP recording from monolayers of substrate-adhered cardiomyocytes (Abbott et al. 2018; McGuire, Santoro, and Cui 2018; Robinson et al. 2012; Xie et al. 2012; Duan et al. 2011; Hai, Shappir, and Spira 2010; Angle, Cui, and Melosh 2015; Spira and Hai 2013; B. X. E. Desbiolles et al. 2019; Benoît X. E. Desbiolles et al. 2020). The recent development of combining vertical nanoelectrodes with CMOS technology has drastically increased the recording throughput of NEAs and has achieved high-throughput recordings in both cardiomyocyte cultures and neuronal networks (Abbott et al. 2020, 2017). Additionally, enabled by integrated current clamp electronics, these methods can now perform intracellular AP recording from Neurons which was previously only achievable by patch clamp (Abbott et al. 2020). NEA devices differ substantially in their shapes, throughput, and mechanisms of gaining intracellular access. Some devices appear to spontaneously gain intracellular access (B. X. E. Desbiolles et al. 2019; Hai, Shappir, and Spira 2010; Robinson et al. 2012; Liu et al. 2017), while others require transient electroporation (Abbott et al. 2017; Lin et al. 2014; Benoît X. E. Desbiolles et al. 2020; Xie et al. 2012; Lin et al. 2017; Abbott et al. 2020) or optoporation (Dipalo et al. 2018, 2021, 2017) of the cell membrane. Although spontaneous intracellular access allows iAP recording, it often occurs randomly with a low probability, and cannot be repeated in a controllable manner (B. X. E. Desbiolles et al. 2019; Benoît X. E. Desbiolles et al. 2020). On the other hand, transient electroporation of the membrane with a short electric pulse is a controlled method of repeatedly gaining intracellular access and recording iAPs at desired time points. Although all NEAs should be compatible with selfreferencing recording, the variable success rate and recording duration have largely prevented their usage for multi-dose drug screening. In addition, the fabrication of nanoelectrodes involves electron beam lithography, which is a costly and serial process. These limitations have so far hindered NEAs from being broadly adopted for drug screening purposes.

Other approaches are being developed as surrogate assays for the measurement of iAPs, including voltage and $\mathrm{Ca}^{2+}$ optical imaging using fluorescent indicators or genetically encoded molecular probes (Broyles, Robinson, and Daniels 2018; Dana et al. 2019; Shroff et al. 2020). These powerful approaches allow simultaneous recording from many cells. That said, some significant drawbacks still exist for optical approaches including the requirement of chemical labeling or genetic modification of cells, photo-toxicity, limited duration of measurement due to photobleaching, and slower on/off kinetics. All these constraints currently prevent them from replacing direct electrophysiological methods for recording iAPs. Rather, it is often desirable to combine electrophysiological and optical interrogation of the same cell for multimodal analysis, such as correlation between iAPs and calcium transients in cardiomyocytes.

Herein, we developed semi-hollow nanocrown electrodes that are mechanically robust and can perform highly reliably and prolonged iAP recordings. Nanocrown electrodes enable parallel, self- 
referencing, and multi-dose drug assessment in hPSC-CMs. When combined with a miniaturized recording apparatus housed inside an incubator, these nanoelectrodes will enable iAP-based drug screening.

\section{Results and Discussion}

\section{Fabrication of robust nanocrown electrode arrays on transparent substrates by photolithography}

Our device fabrication process consists of two parts. In the first part of the device fabrication, we developed a protocol to address issues related to the robustness and the fabrication throughput of vertical nanoelectrode arrays (NEAs). In this protocol, photolithography and a top-down dry etching technique were used to fabricate transparent $\mathrm{SiO}_{2}$ nanopillars $(\sim 1 \mu \mathrm{m}$ in diameter and $\sim 3$ $\mu \mathrm{m}$ in height ) by directly etching into a $\mathrm{SiO}_{2}$ substrate (Fig. 1A). These $\mathrm{SiO}_{2}$ nanopillars are mechanically robust as they are continuous with the substrate at their base. The dry-etching chemistry was optimized to obtain a slightly tapered micropillar profile with a larger diameter and stronger attachment near the base. To reduce the feature size to nanoscale (Lou et al. 2019; W. Zhao et al. 2017), we employed a wet $\mathrm{SiO}_{2}$ etching technique that linearly and uniformly shrinks vertical pillars from $1000 \mathrm{~nm}$ to 500 or even $200 \mathrm{~nm}$ diameter (Fig. 1B). The final diameter is controlled by the duration of the wet etch step. Subsequently, we used photolithography and Pt deposition to pattern conducting electrodes (surrounding the $\mathrm{SiO}_{2}$ nanopillars) and connecting feed lines. The tapered nanopillar favors the metal coverage and continuity at the nanopillarsubstrate edges during metal sputtering. Finally, a thick $600-\mathrm{nm}$ insulating layer of $\mathrm{SiO}_{2} / \mathrm{SiN}_{\mathrm{x}}$ was deposited to insulate the entire surface.

In the second part of the device fabrication, the $\mathrm{Pt}$ and $\mathrm{SiO}_{2} / \mathrm{SiN}_{\mathrm{x}}$-coated nanopillars were fabricated into either nanopillar electrodes or nanocrown electrodes (Fig. 1A). To fabricate nanopillar electrodes, we used a photoresist-masked dry etching step to selectively remove the insulation layer from the vertical nanoelectrodes. To fabricate nanocrown electrodes, the substrate was first coated with a thick and protective photoresist layer followed by a directional dry etching step to remove both the $\mathrm{SiO}_{2} / \mathrm{SiN}$ and the Pt layers from the tip of the nanopillars. Then, the substrate is subjected to a selective and time-controlled wet oxide etching to shorten the $\mathrm{SiO}_{2}$ core and to remove remaining insulating layers from the pillar. Scanning electron microscopy (SEM) images show the shape of nanopillar (Fig. 1C) and nanocrown (Fig. 1D) electrodes. In these images, the crown top of the nanocrown electrode can be clearly identified, with the conductive platinum layer appearing bright while the insulating $\mathrm{SiO}_{2}$ core appears dark.

To accurately examine the depth of the crown top, we used focus-ion-beam (FIB) to vertical mill open nanoelectrodes and then used SEM to image their vertical cross sections. From the FIBSEM cross-section images, the nanopillar electrode shows a uniform platinum coating layer (Fig. 1E), while the nanocrown electrode has a hollow platinum crown 180-200 nm deep for the $3 \mu \mathrm{m}$ - 
tall structure (Fig. 1F). We refer to these electrodes as "nanocrowns" to reflect on their shape and the irregular crown rim. Nanocrown electrodes have a lower impedance than nanopillar electrodes (Fig. 1H), likely due to the crown rim that is exposed to the electrolyte on both sides. Compared to the nanopillar nanoelectrodes, the nanocrown shape will induce the cell membrane to wrap around the outer surface while, at the same time, promotes cell adhesion to the inner core (Figure 1G). This new shape stabilizes the membrane-electrode interface and is important for the repeated and multiple-day recordings. hPSC-CMs were obtained according to our previous protocols (Burridge et al. 2014; Ye et al. 2021). hPSC-CMs cultured on the NEA devices exhibit spontaneous and rhythmic beating after 2-3 days and can be maintained on the devices for three months or longer (Figure 1I).

We used fluorescence imaging to examine cell adhesions to nanocrown electrodes. For this purpose, we transfected U2OS cells with a membrane marker GFP-CAAX and plated them on an NEA device (Figure 1J). Unless nanopillar is mentioned specifically, NEA refers to nanocrown electrode array in the subsequent studies. The cells were fixed and immunostained with antiintegrin beta1 to probe cell adhesions. A bright green dot around the nanocrown electrode indicates that the membrane is wrapping around the vertical nanoelectrodes. The magenta dot around the nanocrown electrode indicates the formation of integrin-containing adhesion complexes. It is worth noting that cell adhesions form on most but not all nanoelectrodes wrapped by the cell membrane. Confocal imaging in the z-direction shows the formation of cell adhesions at the top of the nanoelectrodes (Figure 1K).

As the new fabrication protocol involves photolithography and is wafer-based, the device throughput and reproducibility are significantly enhanced. Twelve NEA chips fit onto a single 4" wafer and more than a hundred NEA chips can be fabricated in a batch (Suppl. Fig. S1). With each chip consisting of 58 recording pads and two stimulation pads, these NEA devices increase the throughput by enabling parallel recordings and screening across many cell cultures and conditions as we show below. Since we used a $\mathrm{SiO}_{2}$ substrate which is effectively a transparent glass surface, these NEAs are compatible with optical imaging and long-term culture of hPSCCMs.

\section{Nanocrown electrodes reliably and repeatedly record intracellular action potentials}

Electrophysiology measurements were carried out 5 to 50 days after cardiomyocytes were seeded on NEAs, when cells were in a confluent monolayer and beat synchronously. Before electroporation, NEAs reside outside the cell membrane and record eAPs with a biphasic spike shape and an average amplitude of about $\sim 1 \mathrm{mV}$, which is similar to that of planar microelectrodes. Upon the application of a single $200-\mu$ s electroporation pulse at $\pm 3.5 \mathrm{~V}$ to a selected nanoelectrode, the recorded action potential transits from an extracellular spike shape to an intracellular waveform (Fig. 2A). In the raw data trace in Fig. 2A, the electroporation event can be easily identified by a sudden rise and temporary saturation of the amplifier. In the same culture, three other nanoelectrodes were simultaneously selected for electroporation. Recordings from these channels showed intracellular recordings while their immediate neighboring channels showed extracellular spikes, indicating that the electroporation and the iAPs were highly localized to the selected nanoelectrodes (Suppl. Fig. S2). 
A NEA device allows simultaneous recording of up to 60 hPSC-CMs. On average, 40-50 electrodes show eAPs and the electroporation-induced extracellular-to-intracellular transition rate is $>99 \%$, i.e. almost all eAP signals are converted to iAPs after electroporation. The amplifier system (Multichannel System, MEA1060) that we use has limited charge injection capacity and usually limits simultaneous electroporation to 4-6 cells. We will discuss a custom-built amplifier system that allows sequential electroporation from all electrodes in the last section of this article. Multiple rounds of manual electroporation applied to different sets of electrodes allow more iAPs to be recorded at the same time. In Fig. 2B, 15 cells' iAPs were recorded simultaneously. While their beating intervals were identical, these iAPs show differences in their amplitudes, upstroke times, and iAP durations. Amplitude-normalized traces show $\sim 49 \mathrm{~ms}$ phase shift in the iAP upstroke time (Fig. 2C). From traces recorded at spatially distinct electrodes, we calculated that the electric signal propagated in the culture dish with a velocity about $0.21 \mathrm{~m} / \mathrm{s}$ (Suppl. Fig. S3). When these iAPs are aligned to their depolarization upstroke with normalized amplitudes, they show distinct waveforms and durations despite beating synchronously (for clarity, 4 of the 15 traces were displayed in Fig. 2D). The differences in waveforms are much larger when comparing iAPs from different cultures that are beating at different frequencies (11 iAP traces from 10 different cultures were shown in Suppl. Fig. S4). The differences in iAP duration, shape, upstroke slope, and repolarization form are indicative of inherent phenotypic variability among hPSC-CMs, which can be greater than drug-induced effects. Therefore, self-referencing of the same cell is important for assessing drug responses using hPSC-CMs.

The extracellular-to-intracellular transition induced by electroporation is due to subnanometersized membrane pores that electrically connect NEAs to the intracellular domain. NEA-induced membrane pores are highly localized and reseal over time (Supp. Fig. S5). Modeling of the electric fields shows that, during the electroporation step, the electric field is significantly enhanced at the rim of nanocrowns (Suppl. Fig. S6). To probe the size of the membrane pore, we monitored the cellular entrance of calcein, a method often used to probe membrane integrity. Calcein is a membrane impermeable fluorophore about $1 \mathrm{~nm}$ in size. We found that calcein was able to get into cells when added 1 min before electroporation, but not when added 1 min or longer after electroporation (Suppl. Fig. S7). This result suggests that the membrane pore has resealed to be less than $1 \mathrm{~nm}$ at 1 min after electroporation, when our NEA recording of iAPs usually starts. Therefore, these membrane pores do not cause leakage of cellular content and do not perturb cell physiology (more discussions later).

We carried out systematic measurement of daily eAP and iAP measurements from 16 cells over a period of 12 days using multiple cultures (Fig. 2E and Suppl. Fig. S8). At each date, the culture was first measured for eAP recording, and then electroporated for iAP recording before being returned back to the incubator. The high quality eAP and AP signals over the time course of 12 days indicate that daily electroporation did not perturb the cell health. Additionally, the consecutive daily measurements do not change the amplitude of recorded eAPs or iAPs over the 12 days period (Figure 2F). In Suppl. Fig. S9, we show recordings from a cell that was repeatedly electroporated and measured for 15 times from day 3 to day 43 after being seeded on the device. Nanocrown electrodes are mechanically robust because their $\mathrm{SiO}_{2}$ cores are continuous with the 
bulk substrate. The nanocrown NEA devices can be repeatedly cleaned and re-used for many cycles of cardiomyocyte cultures. Suppl. Fig. S10 shows the recordings from the same device in six independent cultures over a year. The device performance (signal amplitude and noise) is stable over repeated usage. This remarkable signal stability and device robustness afforded by nanocrown electrodes are not observed using other types of NEA devices.

Our results indicated that the depth of the crown significantly affected the electrode performance. In our fabrication process, the depth was controlled by the $\mathrm{SiO}_{2}$ core etching time. We fabricated nanocrowns of two depths and systematically compared the performance of nanopillar electrodes, 450-nm-deep ( 10min etching time) nanocrown electrodes, or 180-nm-deep ( 3 min etching time) crowns. For this study, we recorded 76 cells using nanopillar electrodes, 111 cells using 450-nm deep nanocrown electrodes, and 308 cells using 180-nm-deep nanocrow electrodes for 5 mins. These measurements were from many independent cultures and pooled together for statistical analysis. Both 450-nm-deep and 180-nm-deep nanocrown achieved $>99 \%$ success rate for electroporation-induced extracellular-to-intracellular transition, which was better than the $94 \%$ for solid nanopillar electrodes (Fig. 2G). The iAP amplitude measured by $180-\mathrm{nm}$-deep nanocrown electrodes $(21.76 \mathrm{mV}, \mathrm{N}=308)$ is twice as large as that measured by $450-\mathrm{nm}$-deep nanocrown electrodes (9.7 mV, $\mathrm{N}=111)$ or by nanopillar electrodes (10.7 mV, $\mathrm{N}=76$ ) (Fig. 2H). When we compared the time-dependent decay of iAPs, $100 \%$ of the $180-\mathrm{nm}$-deep nanocrown electrodes maintained intracellular access at the end of 5-min recording, as compared to $80 \%$ for $450-\mathrm{nm}$ deep nanocrown electrodes and 70\% for nanopillar electrodes (Fig. 2I). In another experiment, longer 15-min recordings were performed using the 180-nm-deep nanocrown electrodes $(\mathrm{N}=41)$. An impressive $100 \%$ of these recordings maintained intracellular at the end of 15 -min recordings, which was rare for nanopillar electrodes. This prolonged intracellular access is crucial for pharmacological experiments as we show in the next section. The better performance of the 180nm-deep crown is likely due to the cell membrane forming more stable adhesions to the inner $\mathrm{SiO}_{2}$ core than that for 450-nm-deep nanocrowns (Fig. 2J). The 180-nm-deep nanocrown electrodes are used for the following studies.

\section{Simultaneous NEA and Patch recordings from the same cell confirm that NEAs record accurate iAP waveforms}

To determine the accuracy of iAP waveforms recorded by NEAs, we compared these measurements with those recorded by manual patch clamp, which is the gold standard of intracellular recording. In these experiments, we performed simultaneous patch clamp and NEA recordings on the same cell (Fig. 3A). The transparent substrate allowed us to identify hPSCCMs in contact with NEAs under a microscope for manual patch clamping (Fig. 3B). Upon obtaining a high seal resistance with the patch pipette, suction was applied through the pipette to breach the membrane and thus allowing iAPs to be recorded by the patch electrode. Before applying the electroporation pulse at $t=0$, the patch electrode recorded typical iAP waveforms, while the simultaneous NEA electrode recorded characteristic eAP spikes (Fig. 3C-D). After NEA electroporation, both the patch and the NEA electrodes showed intracellular AP waveforms, albeit with different amplitudes (Fig. 3D). The electroporation event showed up as a sudden rise and saturation of amplifiers in both NEA and patch traces, which was used to align the two time 
traces. We note that electroporation-induced decrease of patch iAPs is due to a reduction in the patch sealing resistance, not a change of the resting membrane potential. Sudden decrease of patch iAP amplitude due to loss of sealing resistance is common in patch recordings from cardiomyocytes that were not cultured on nanoelectrodes (Suppl. Fig. S11).

We wrote software to automatically calculate a single scaling factor for each pair of patch- and NEA-recorded iAPs to account for the difference in their amplitudes. The scaled and overlaid iAP traces show that the two waveforms almost entirely overlap (Fig. 3E). We carried out the simultaneous patch and NEA dual recordings for a total of 10 cells, all showing near-perfect overlap after scaling (see Suppl. Fig. $\mathbf{S 1 2}$ for 8 more examples of dual-recording traces). From iAPs recorded by NEA and patch, we calculated a set of parameters known to be related to druginduced proarrhythmia (Fig. 3F). These parameters included cycle time, diastolic interval (time interval where the membrane potential is more negative than $90 \%$ repolarization), action potential durations APD90 and APD50 (time taken to reach $90 \%$ and $50 \%$ of repolarization, respectively), and t_rise (the depolarization time from APD80 to APD20). Despite large variations among individual cells for each parameter, recordings of Patch and NEA from the same cell agree nearly perfectly with each other (Fig. 3G). Averaging over the 10 experiments, the differences between NEA and patch measurements were minimal: $0.05 \pm 0.08 \%$ for cycle time, $0.8 \pm 0.6 \%$ for APD90, and $3.21 \pm 2.10 \%$ for APD50, $0.85 \pm 0.65 \%$ for diastolic intervals, and $6.67 \pm 9.75 \%$ for $t$ rise (Fig. 3I). The comparison of simultaneous Patch and NEA recordings confirm that NEAs provide accurate measurement of iAP waveforms and parameters.

\section{NEA records accurate iAP waveforms despite time-dependent decrease of the iAP amplitude}

Resealing of the electroporation-induced membrane pores over time causes the amplitude of NEA-measured iAPs to decrease over time. In the trace shown in Fig. 4A, the NEA iAPs decrease by an order of magnitude from $14 \mathrm{mV}$ at $60 \mathrm{~s}$ to $1.3 \mathrm{mV}$ at $530 \mathrm{~s}$ after electroporation. In the same time period, the patch iAPs from the same cell first increased and then exhibited large fluctuations after $430 \mathrm{~s}$. The zoom-in plots show 15-s windows at three time points, $t=0,80$, and $430 \mathrm{~s}$, for both patch and NEA traces (Fig. 4B). From the patch trace around $t=0 \mathrm{~s}$, we can clearly see that the amplitude of the patch iAP is reduced from $95 \mathrm{mV}$ to $35 \mathrm{mV}$ upon electroporation at $\mathrm{t}=0 \mathrm{~s}$. Despite the drastic change in the patch iAP amplitude, the cycle time is unperturbed and the overall iAP waveform remains the same. The NEA trace shows extracellular spikes before electroporation and then is out of range immediately after electroporation because of the amplifier saturation. In the $t=80$ s zoom-in window, the NEA electrode has recovered and both patch and NEA electrodes record iAPs that are synchronized in time and show similar waveforms but with different amplitudes. In the $t=430$ s zoom-in window, the patch iAP shows a sudden decrease in amplitude at $t=432 \mathrm{~s}$, likely due to a decrease of the patch sealing resistance. The event shows as a slight change of the baseline in the NEA channel without changing the iAP amplitude or the waveform.

We developed a software package that automatically detects the spiking point (red stars), the starting point (green stars), the maximum point (blue stars), and the minimum point (cyan stars) 
of each iAP in the entire trace. A few iAPs immediately after the electroporation are omitted from analysis as there are often spurious artifacts. From these identified points, the software calculates the time-dependent evolution of cycle time, diastolic interval, APD90, APD50, amplitude, and spike velocity (dV/dt). Detailed analysis of traces shown in Figure 4A is presented in Fig. 4C. Detailed analysis of traces shown in Figure 3C is included in Suppl. Fig. S13. The analysis results show that the cycle time and the diastolic interval remain the same before and after electroporation despite the dramatic change of the amplitude. Therefore, the physiological functions of cardiomyocytes are not perturbed by NEA-delivered electroporation. APD90 and APD50 are slightly decreased immediately after electroporation but remain stable afterward. For the NEA trace, APD90 and APD50 remain constant for the entire time despite an order of magnitude decrease in amplitude. On the other hand, the amplitude and the spike velocity, which is dependent on the amplitude, show large changes. We note that, when the electroporation pulse is delivered simultaneously to many NEA pads, e.g. $>30$, it sometimes stimulates the whole culture and reduces the cycle time regardless of whether a cell is electroporated or not.

To further confirm that the patch and NEA iAP waveforms are not dependent on their amplitudes, we presented the scaled and overlaid iAPs at four different time points (Figure. 4D). During the time period, the patch iAP amplitude increased from $71.81 \mathrm{mV}$ to $92.68 \mathrm{mV}$ and then decreased to $81.39 \mathrm{mV}$. The NEA iAP amplitude decreased by about an order of magnitude from $12.76 \mathrm{mV}$ $(\mathrm{t}=80 \mathrm{~s}), 5.17 \mathrm{mV}(\mathrm{t}=300 \mathrm{~s}), 2.28 \mathrm{mV}(\mathrm{t}=430 \mathrm{~s})$, and $1.36 \mathrm{mV}(\mathrm{t}=530 \mathrm{~s})$. Despite their dramatic differences in amplitudes, the pairs of iAPs at the same time overlay nearly perfectly. The APD90 deviation between paired Patch/NEA iAPs (Fig. 4E) and the overall waveform deviation (Figure. 4F) are $\sim 1 \%$ for the entire period, which is negligible as compared to the drug effects we show later. From a simplified circuit model (Suppl. Fig. S14), both patch and NEA signals are linearly dependent on the biological sigal, which explains why they report the same iAP waveform despite differences in amplitudes.

\section{NEA enables self-referencing assessment of drug-induced changes of iAPs}

As shown in Fig. S4 and in previous studies (Zhu et al. 2016), hPSC-CMs are mixed populations with cells showing heterogeneous iAP waveforms. Furthermore, the iAP waveform is affected by the cell density, beating frequency, and culture conditions (Du et al. 2015). The variability among iAPs underlied by phenotypical and physiological differences between cells and cultures can be much larger than drug-induced effects on a given cell's iAP. This necessitates self-referencing comparing the before-drug and after-drug iAPs in the same cell. Indeed, the standard patch clamp-based drug assay is self-referencing. However, the patch clamp method is labor intensive, low throughput, and not suitable for drug screening in hPSC-CMs.

NEAs enable self-referencing measurements of drug effects in parallel. For this study, we used NEAs to test a number of compounds with known arrhythmogenic risk selected from the CiPA library (Strauss et al. 2019; Colatsky et al. 2016). After electroporating and obtaining stable iAP signals, we first added DMSO as a vehicle compound (Fig. 5A). Subsequently, with continuous iAP recording, four increasing doses of a selected drug (dissolved in DMSO) were sequentially added to the cell culture every 3-4 minutes $(0.3 \mathrm{nM}, 1 \mathrm{nM}, 3 \mathrm{nM}, 10 \mathrm{nM}$ of dofetilide for the trace 
shown in Fig. 5A). The mechanical agitation caused by liquid exchange induced signature fluctuations both in the measured channel and in the non-electroporated control channels, which we used to indicate the drug addition time. In each trace, 1000-2000 intracellular APs were recorded during the 15-20 min recording period (Fig. 5A).

Using self-referencing NEA recordings, we compared a group of common high, low and medium risk compounds that are known to distinctly alter the shape of iAPs (Fig. 5B). High-risk compounds such as dofetilide and D,L-sotalol are known to prolong iAP durations, while low-risk compounds such as verapamil and nifedipine reduce iAP durations (Marschang et al. 1998; Bayer et al. 1977; Kimura et al. 1992). To examine drug effects, action potentials were selected for analysis 3-min after each dose addition. Action potentials from the same cell were normalized and aligned to their depolarization phase. By self-referencing, drug-induced prolongation of the action potential duration is clearly visible for dofetilide and D, L-sotalol (Fig. 5B). On the other hand, drug-induced shortening of the action potential duration is evident for verapamil and nifedipine (Fig. 5B). Another compound, cisapride induced early-afterdepolarization events. These data shows that NEAs are able to reliably capture drug-induced effects by self-referenced recordings.

Interestingly, after analyzing many cells, we found three types of dose responses to Dofetilide. For the first type, increasing doses of dofetilide at $0.1,0.3,1,3,10 \mathrm{nM}$ cause a monotonic dosedependent increase of APD90 as shown in the first APD90 trace in Fig. 5C, which represents $\sim 50 \%$ of cells. For the second type such as the second APD90 trace in Figure 5C that represents $30 \%$ of cells, the highest dose caused shortening of APD90, i.e. refractory dosage at $10 \mathrm{nM}$. For the third type, the medium dose of $3 \mathrm{nM}$ already caused shortening of APD90, i.e. refractory dosage at 3 nM. Fig. 5D shows 3s zoom-in windows of the raw traces at 60s, 600s, and 600s. The iAPs for the first type get wider with increasing drug doses, but the fast uprising of the depolarization phase remains similar. For the second and the third types after the refractory dosage, $10 \mathrm{nM}$ for the second type and $3 \mathrm{nM}$ for the third type, the iAP duration gets narrower but the shape is drastically different from the narrower iAPs at lower doses. In particular, the uprising duration is longer and the overall waveform is more triangularized. For cell 2, some action potentials are missing after $900 \mathrm{~s}$.

Quantification of APD90, APD50, t_rise, and triangulation (APD90-APD30) highlights the variabilities of drug-induced responses in different cells and the importance of self-referencing in identifying these variations (Fig. 5E). The unique advantage of NEAs for drug assessment is that one can measure iAPs at multiple drug doses from the same cell and measure the responses of multiple cells in the same experiment. In this way, a set of dose-response data can be obtained with self-referencing to avoid the large cell-to-cell and culture-to-culture variations inherent to hPSC-CMs. APD90 and APD50 are often used for assessing drug effect. In a couple of days, we were able to measure 84 self-referencing dose-response curves using independent cultures (Fig. $5 \mathrm{E})$. Membrane pores on some of these cells were sealed before higher doses were added, so there are fewer data points for higher doses, for example, $\mathrm{N}=84$ for the control and $\mathrm{N}=40$ for the highest dofetilide concentration at $10 \mathrm{nM}$ (Fig. 5E). Nevertheless, the $\mathrm{N}=40$ per dose sample size is already significantly larger than previous studies reporting iAP dose-response measurements 
with $\mathrm{N}<10$. From the APD90 plot, it is obvious that some cells show monotonic dose-dependent increase, while others show refractory dosage at $3 \mathrm{nM}$ or $10 \mathrm{nM}$. It is interesting that, for cells that show decreased APD90 after the refractory dosage, their t_rise parameter shows significant increase (Fig. 5E).

Unlike patch clamp or other invasive iAP recording methods in which the cells are no longer viable after recording, the NEA measurements are minimally invasive. Therefore, after washing out drugs and allowing 1-2 days of recovery in the incubator, the same hPSC-CM culture can be used again for assessment of the same or different drugs. In one experiment, we showed the dofetilide dose-responses from the same culture at day 1, day 3, and day 4, with the three independent sets of experiments showing slightly different drug effects induced by dofetilide (Suppl. Fig. S15). The fact that the same hPSC-CM culture can be used repeatedly for multiple drug assessment experiments reduces the number of precious hPSC-CM cell cultures required for obtaining doseresponse curves, thereby facilitating the drug screening process.

\section{In-situ recording using miniaturized Petri-dish-like recording stage}

All the data shown earlier were collected using a commercially available amplifier (MEA 1060, MCS), which requires that the culture be taken out of the incubator and placed on the recording stage for electrophysiology measurements. The health and the electrophysiological properties of cardiomyocytes are sensitive to experimental conditions such as temperature, humidity, and $\mathrm{CO}_{2}$ level (Kohlhardt and Haap 1976; Cedrini and Alloatti 1979). To reduce environmental variabilities, we recently collaborated with Cyion Technologies to design a miniaturized recording stage for in situ long term recording inside a $\mathrm{CO}_{2}$ incubator, which is controlled through a cable connected to a computer outside the incubator. The printed circuit board hosting our NEA devices was fitted with a bottomless $35 \mathrm{~mm}$ Petri-dish (Fig. 6A-B). In this design, the Petri-dish is placed on the recording stage with a short distance between the cell and the amplifier to minimize signal loss or distortion (Fig. 6C).

The new Cyion amplifier enabled eAP recording, electroporation, and AP recording inside an incubator (Fig. 6D), in ways similar to that described earlier. In situ recording significantly reduced temperature-dependent variations in the cells' electrophysiological behavior. Immediately after a culture was taken out of the incubator and mounted on a recording stage, we often observed a significant change in the cycle time, which is known to be affected by temperature (Cedrini and Alloatti 1979) (Fig. 6E). By comparison, the cycle time was much more stable when using our custom recording stage housed inside the incubator. The Cyion amplifier stage also supports automated sequential electroporation of all NEA channels and therefore enables simultaneous iAP recording of up to 60 channels (Fig. 6F). Therefore, the miniaturized Petri-dish-like recording stage is ideally suited for iAP recordings. These new capabilities further enhance the advantage of using NEAs for preclinical evaluation and screening of new drug candidates in hPSC-CMs.

\section{Conclusions}


Herein, we presented the development of semi-hollow nanocrown electrodes. The crown shape induces the cell membrane to wrap around the outer surface and to adhere to the inner core, which stabilizes the membrane-electrode interface. Nanocrown electrodes achieve 99\% electroporation success rates and reliably measure iAPs from cardiomyocytes with high accuracy and signal strength. The prolonged recordings allowed us to perform a series of pharmacology experiments based on the self-referencing iAP waveform. The development of a Petri-dish NEA and an accompanying miniaturized recording apparatus would enable in situ recording of iAPs inside $\mathrm{CO}_{2}$ incubators. These developments will hopefully enable iAP-based assays for dosedependent drug screening.

\section{Methods}

\section{Nanopillar and Nanocrown electrode array fabrication}

Quartz wafers were cleaned and deposited with HMDS to promote resist adhesion. A positive photoresist (Shipley 3612) was spin-coated and exposed by a maskless aligner (Heidelberg MLA150) to pattern circular holes. A Cr mask of $120 \mathrm{~nm}$ was later deposited by metal evaporation before being lifted-off in acetone to make circular Cr disks. Using the deposited $\mathrm{Cr}$ as a mask, vertical microstructures were subsequently generated by anisotropic reactive ion etching with a mixture of C4F8, CHF3, and Ar (Versaline LL-ICP Oxide Etcher) to precisely control the vertical profiles of the quartz nanostructures. The substrates were then immersed in Chromium Etchant 1020 (Transene) to remove the chrome mask and subsequently wet-etched in Buffered Oxide Etchant (BOE) 20:1 (Transene) to decrease the nanostructures' dimensions. For electrode fabrication, a bilayer resist strategy was used to construct metal connection lines with smooth edges. Microposited LOL and Shipley 3612 were spin-coated, baked, and then exposed to create an undercut in the Microposited LOL layer underneath the resist for the lift-off step. A layer of $\mathrm{Ti}$ $(10 \mathrm{~nm})$ and Pt (40 nm) was sputtered and then lifted-off in Microposit Remover 1165 for 16 hours. Subsequently, the insulation layers composed of $60 \mathrm{~nm}$ of Si3N4 and $540 \mathrm{~nm}$ of SiO2 were deposited via plasma-enhanced chemical vapor deposition ( $350{ }^{\circ} \mathrm{C}$; Plasma-Therm Shuttlelock).

For nanopillar electrodes, the insulation layer was removed from the nanopillars through the spin coating of a thin layer of resist, followed by a wet etch in Buffered Oxide Etchant 20:1 (Transene). For nanocrown electrodes, a hollow conversion strategy was applied after the insulation step to create nanocrowns. Briefly, a layer of photoresist was spin-coated to protect the entire substrate. Then, the substrates were anisotropically etched with Ar (MRC model $55 \mathrm{RIE}$ ) to remove the insulation layer and the metal layer at the tip of the nanostructures and expose the inner quartz. The inner quartz core was subsequently etched with BOE The depth of the nanocrown was controlled by varying the etching time between 3 to 10 minutes. Nanocrowns with $450 \mathrm{~nm}$ or 180 $\mathrm{nm}$ crown depths were fabricated by $10 \mathrm{~min}$ or $3 \mathrm{~min}$ BOE etching respectively. Finally, the quartz wafers were diced into $12.5 \mathrm{~mm} \times 12.5 \mathrm{~mm}$ pieces by the Micro Dicing Services to fit our custom made printed circuit boards. The final devices were characterized by SEM (FEI Nova).

\section{hPSC-CM differentiation.}


hPSC-CMs were differentiated and purified based on our previous publications (Wu et al. 2019; Burridge et al. 2014; M.-T. Zhao et al. 2017) Briefly, hPSCs with 90-95\% confluency were treated with $6 \mu$ M CHIR99021 (Selleck Chemical) in RPMI (Gibco®, Life Technology) supplemented with B27 w/o insulin for 2 days, and allowed to recover for 1 day in RPMI plus B27 without medium. Then cells were treated with $5 \mu \mathrm{M}$ IWR-1 (Selleck Chemical) for 2 days. After recovering in the fresh RPMI plus B27 without insulin medium for another 2 days, cells were switched to RPMI plus B27 with insulin for 2 days. Beating hPSC-CMs were derived around 9-11 days after differentiation. The hPSC-CM were purified with glucose-free RPMI plus B27 with an insulin medium for 2-4 days. Differentiated hPSC-CMs were maintained in RPMI plus B27 with insulin medium for the following experiments.

\section{Cell Culture of hPSC-derived-CMs on NEA devices}

hPSC-CMs were maintained in Matrigel pre-coated 6-well plates in RPMI 1640 medium (Gibco REF 11875-093 500ml) with B-27 supplement (Gibco REF 17504-044 10ml). The culture medium was refreshed every 2-3 days. Before seeding cells on NEA devices, the devices were coated with $1 \mathrm{mg} / \mathrm{ml}$ poly-L-Lysine at room temperature for $15 \mathrm{~min}, 0.5 \%$ Glutaraldehyde in PBS at room temperature for 10min, and followed by 1:200 Matrigel (Corning REF 356231) in DMEM/F12 (Gibco REF $10565-018$ ) at $37^{\circ} \mathrm{C}$ for $3 \mathrm{~h}$ or overnight. A three times PBS wash was performed between each coating step. Cells were dissociated with TryPLE select 10X (Gibco REF A1217701 ) at $37^{\circ} \mathrm{C}$ for $5 \mathrm{~min}$ and $\sim 12 \times 10^{5}$ cells were plated in each coated device in culture medium supplemented with 10\% KnockOut Serum Replacement (KSR)(Gibco REF A31815-01), and cultured in a $5 \% \mathrm{CO} 2$ incubator at $37^{\circ} \mathrm{C}$. The medium was refreshed with normal culture medium every 1-2 days from the second day after replating. Measurements were done 5-30 days postplating unless otherwise stated. The devices are reusable after the following washing steps in the device wells: 5 min of incubation with TrypLE Express (Gibco REF 12605-028) at $37^{\circ} \mathrm{C}$ to remove cells, overnight incubation with Enzymatic cleaner (Ultrazyme) in PBS at room temperature, and leave in $70 \%$ ethanol for at least $1 \mathrm{~h}$. The wells are always immersed in $70 \%$ Ethanol until the next usage. The devices were dried of ethanol and further decontaminated with UV illumination for 30 min before coating for the next cell culture.

\section{NEA-Patch-Clamp simultaneous Recording}

Simultaneous patch-clamp and NEA recordings were carried out on hPSC-CM at room temperature in the culture medium (RPMI $1640+$ B-27). For patch-clamp, the action potential was recorded under whole-cell current-clamp mode using Multiclamp 700B amplifier and Digidata 1440A digitizer (Axon Instrument) with pClamp10 software (Molecular Device). Borosilicate patch pipettes (Sutter Instruments, O.D.: 1.5mm, I.D.: 0.86mm) were pulled with a P1000 pipette puller (Sutter Instrument) with resistances between 2 and $6 \mathrm{M} \Omega$. Pipette internal solution contains in mM: $120 \mathrm{~K}$ Aspartate, $20 \mathrm{KCl}, 1 \mathrm{MgCl}_{2}, 5 \mathrm{EGTA}-\mathrm{KOH}, 0.1 \mathrm{CaCl}_{2}, 10 \mathrm{HEPES}, 4 \mathrm{Mg}_{2} \mathrm{ATP}$. The pH was adjusted to 7.20 with $\mathrm{KOH}$. The patch-clamp recording was done at $10 \mathrm{kHz}$ sampling rate and a $10 \mathrm{kHz}$ Bessel filter was applied. During the experiments, cells occupying NEA pads were located through a microscope. The extracellular signal recording was initiated on NEA followed by patching of the cell. After action potential signal was seen through the patch-clamp, electroporation was applied to the same cell through nanoelectrodes to get the intracellular signal. 
Recordings from both NEA and patch electrodes started before the electroporation and continued during and after the electroporation. Data analysis was performed with Matlab and Python as stated below.

\section{Electric field models}

To assess the difference in electric field strength between hollow and full nanopillars, we performed a finite-element analysis of the voltage distribution in $3 \mathrm{D}$ in vacuum. We did not simulate the electrolyte and double-layer effects for simplicity. Therefore, the obtained results can qualitatively translate only to the electric field strength during fast transients when operating the nanopillars in electrolytes. We used FEniCS, a Python implementation of the finite element method which allows simulating partial differential equations in complex geometries.

Exploiting the radial symmetry of the problem, the simulation reduces to $2 \mathrm{D}$ by simulating on a plane through the pillar's center. The full nanopillar is simulated as a trapezoid to enable simulating angled wall profiles. The hollow nanopillar consists of the same trapezoid where the outer edges are thickened to a given value and the top edge is removed.

The weak form of Laplace's equation in vacuum with no source term is solved on the domain which consists of a square that is 10 times the height of the nanopillar in every dimension in order to minimize numerical edge effects. The boundary conditions are 0 Volt on the box edge, except for the bottom of the nanopillar. Every edge of the nanopillar is set to 1 Volt.

The electric field is computed by taking the gradient of the resulting voltage field in the $\mathrm{x}$ and $\mathrm{y}$ directions. The magnitude of the electric field is obtained by summing the square of the $x$ and $y$ components of the electric field. By interpolating the magnitude of the field spatially, we can compare the relative electric field magnitudes between the full and hollow nanopillars at the same locations (Fig. S8). The code to generate the voltage and electric field distributions based on the FEniCS Python package is available at [DATA_AVAILABILITY_REPO] in the form of a Jupyter Notebook.

\section{Analysis of iAP data}

All data analysis was automated and free of manual intervention. From a raw iAP trace recorded by either NEA or patch electrode, its corresponding standard deviation trace is calculated using a 1-ms sliding window. A peak finding algorithm subsequently identifies the peaks from the standard deviation trace, which correspond to the sharpest-rising points of individual iAPs. Then, the software examines the raw data trace to the left of each rising point to identify the starting point of each IAP, and to the right of each rising point to identify the maximum point of each iAP. The amplitude is calculated from the starting point to the maximum point. APD90 and APD50 are calculated as the width of the iAPs at 90 and 50 percent of repolarization. T_rise is the time that it takes from the 20 percent to the 80 percent of depolarization.

For scaling Patch and NEA recorded iAPs, the two recording traces are first time-aligned to the electroporation time and resampled to the same frequency of $2 \mathrm{kHz}$. Then, each trace is separately processed to identify the rising points of all iAPs. The software then automatically calculates a scaling factor for each pair of iAPs to account for their differences in amplitudes. This 
is done using the mean square error algorithm by first setting the mean of iAPs to 0 and normalizing the amplitude of the patch iAP to 1. Then, the scaling factor for the NEA iAP is calculated as sum(iAP2. $\left.{ }^{\star} \mathrm{iAP} 1\right) / \mathrm{sum}\left(\mathrm{AP} 2^{\wedge} 1\right)$. After scaling, the deviation of the two iAP waveforms is calculated as the mean absolute deviation.

\section{Pharmacology experiments}

All pharmacology experiments were conducted using the following procedure. First, 4-6 cells were electroporated and iAP signals were obtained and recorded until a stable baseline was reached for all signals. In order to obtain consistent recording files for all pharmacology experiments (such as the one shown in Fig. 5A), after the stable iAP baseline was reached, a new recording file was then started and continuously recorded for $30 \mathrm{~min}$. At $20 \mathrm{~s}$, DMSO was added as a control, followed by a sequential addition of increasing doses of the selected drug at $80 \mathrm{~s}, 280 \mathrm{~s}, 460 \mathrm{~s}$ and $640 \mathrm{~s}$ (as shown in Fig. 5A).

Data analysis for Pharmacology experiments. Individual peaks were detected at and around time $75 \mathrm{~s}$ for the control data, and at $260 \mathrm{~s}, 440 \mathrm{~s}, 625 \mathrm{~s}$, and $815 \mathrm{~s}$ for the four drug doses. The three action potentials closest to these timepoints were used for analysis. Differences in APD values between these three action potentials were usually under $1 \%$. If AP signals at a low drug dose started to exhibit extracellular signatures, the data will not be analyzed for higher drug doses. To overlay the iAPs at different drug doses, we time-aligned the traces to their sharp rising point with normalized amplitudes.

\section{Acknowledgements}

This work is supported by National Institutes of Health (R01GM125737 (Cui), R35GM141598 (Cui), R01HL155282 (MTZ), R01HL145676 (JCW), and UH3TR002588 (JCW)), American Heart Association (AHA) Career Development Award 18CDA34110293 (MTZ), and Google LLC. award (ZJ and Cui). 


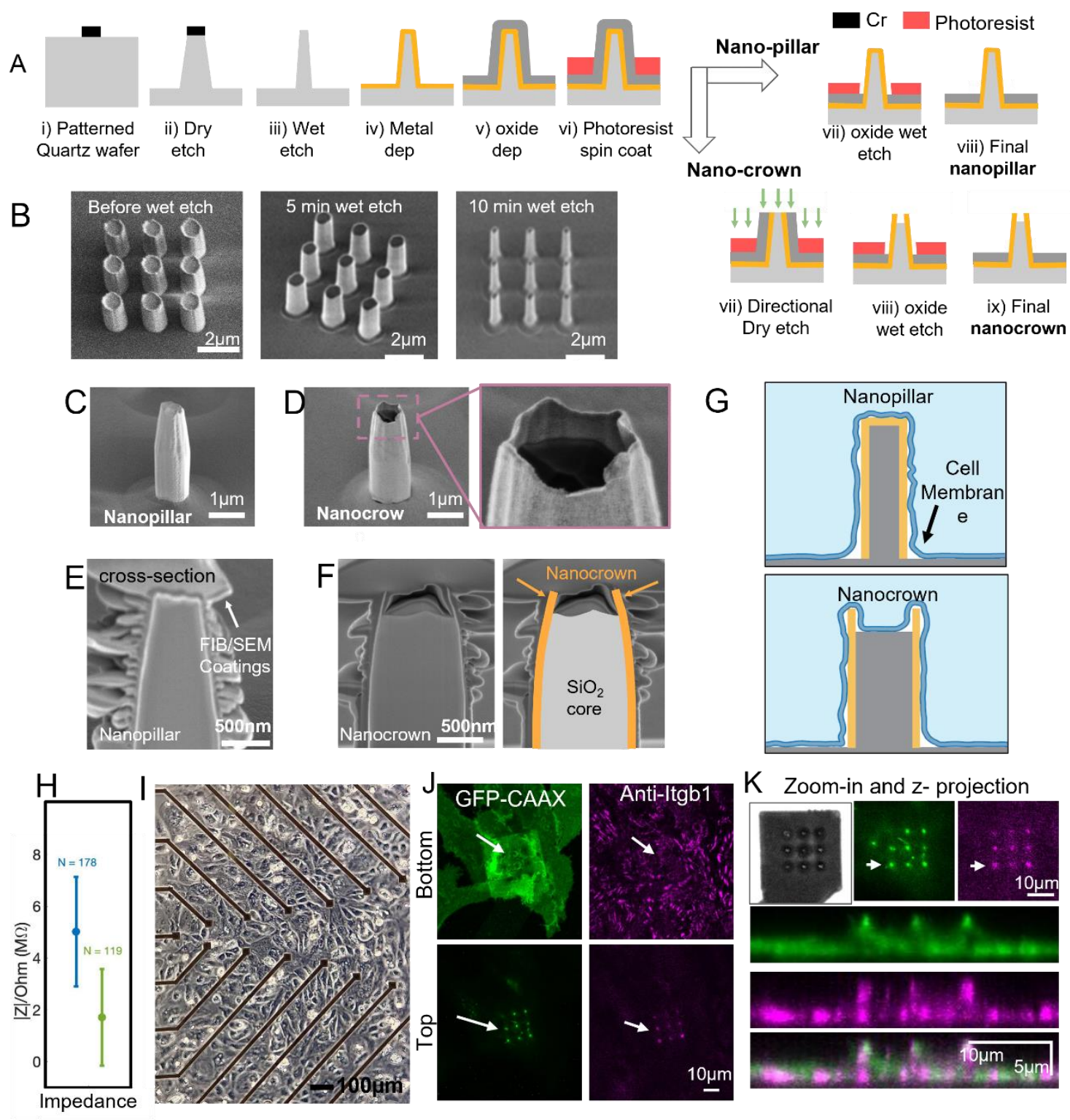

Figure 1. Fabrication and characterization of robust nanopillar and nanocrown electrodes arrays (NEAs) by optical lithography. A) Illustrations of the nanofabrication steps for NEAs. B) SEM images of fabricated pillars before and after thinning with 5-min and 10-min BOE wet etch. C) SEM image of a nanopillar electrode. D) SEM image of a nanocrown electrode. E) SEM image of a Focused ion beam (FIB) milled cross section of a nanopillar electrode. F) FIB/SEM cross-section image of a nanocrown electrode (the Pt metal crown is outlined in orange and the SiO2 in grey). G) Schematic illustration of the interface of nanopillar and nanocrowns with the cell membrane. The membrane wraps around outside of the nanocrown electrode as well as adhere to the $\mathrm{SiO}_{2}$ core. $\mathrm{H}$ ) Impedance of nanopillar (blue) and nanocrown (green) NEAs at $1 \mathrm{kHz}$. I) Brightfield microscope image of a monolayer of iPSC-CM cells on a NEA device. J) Confocal images of a cell on a nanocrown electrode, focused on either the top or bottom of the nanocrown. The cell membrane (CAAX:green) and Integrin adhesion proteins (Anti-Itgb1:purple) can be seen on top of the nanocrowns (white arrows show location of nanocrowns). K) Higher magnified image, and a z projection of a cell on top of nanocrown electrodes showing the membrane (green) and integrins (purple) projection along the nanocrown height. 
A

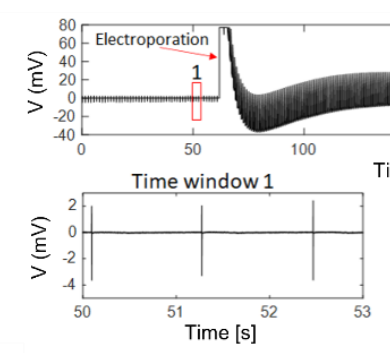

$\mathrm{B}$

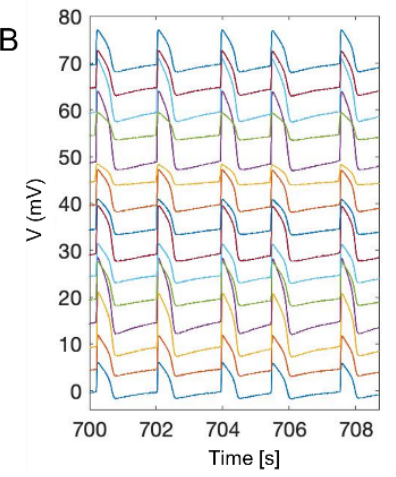

2
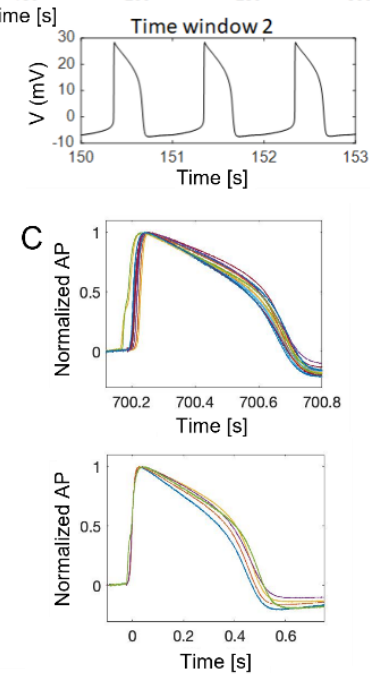

$E$

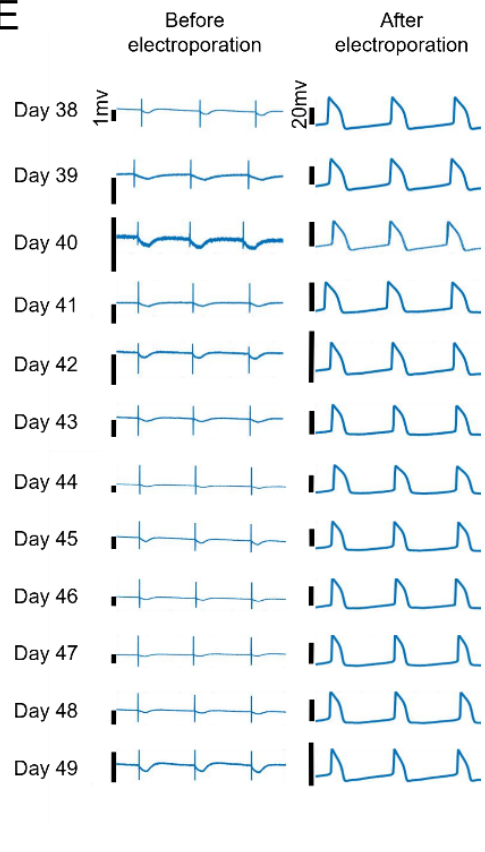

$\mathrm{F}$

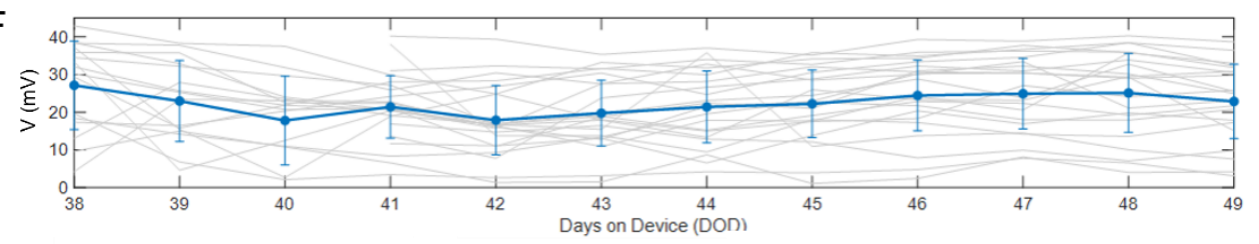

G
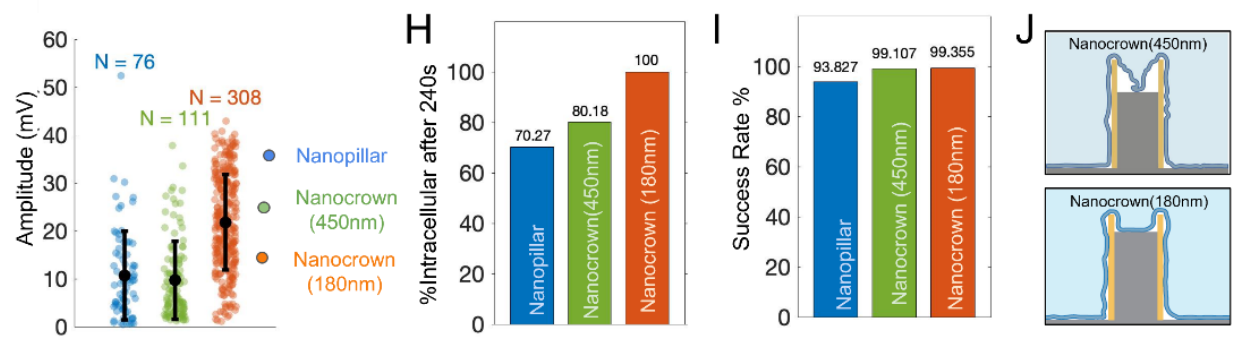

Figure 2. NEAs enable reliable and noninvasive recording of intracellular action potentials (iAPs). A) A representative raw trace of NEA recording from a hPSC-CM. NEAs measure eAP spikes before electroporation (time window 1), and high amplitude iAPs waveforms after electroporation (time window 2). B) Simultaneous iAP recordings from 15 NEA channels show synchronous beating in the same culture. The 9-second traces are vertically shifted for clarity. C) Overlay of amplitude-normalized iAPs illustrates 50-ms phase shift in depolarization time. D) Five of the 16 iAPs are aligned to their rising times. They show distinct iAP durations. E) Twelve recording sessions of eAPs and iAPs from the same cells over two weeks in culture. The recording session started at day 38 on device. For each recording session, eAPs were first measured for $1 \mathrm{~min}$ before an electroporation pulse was applied, which converted the signal to iAPs. F) The average amplitudes of recordings on the same cells indicating a stable signal amplitude with daily-repeated recordings. G) The initial amplitudes of iAPs measured by Nanopillar electrodes (blue), Nanocrown electrodes with 450nm depth (green) and Nanocrown electrodes with 180nm depth (Orange). G) Electroporation success rates for Nanopillar and Nanocrown electrodes. I) Percent of cells that maintained intracellular access at the end of the recording ( 240s after electroporation). J) schematic representation of the interface of the cell membrane and the nanocrown electrodes with $450 \mathrm{~nm}$ and $180 \mathrm{~nm}$ crown depth. 
A

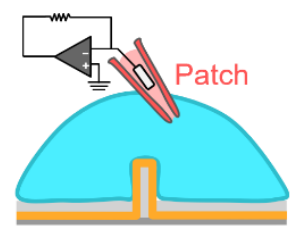

B

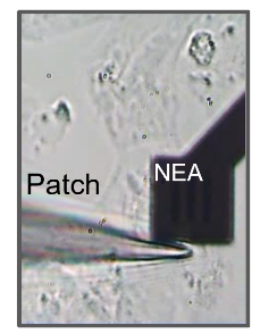

C

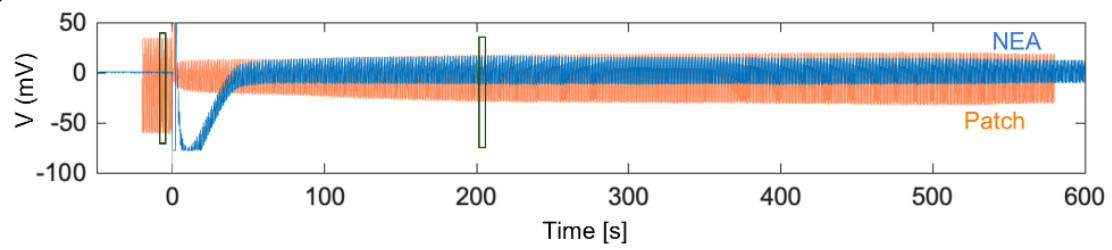

D
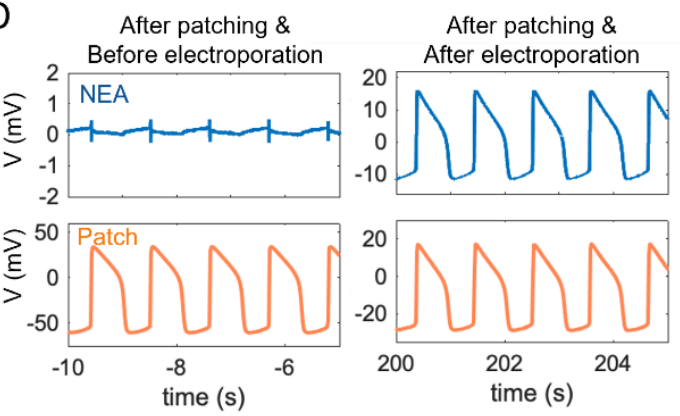

E

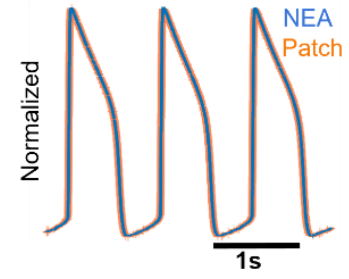

$\mathrm{F}$

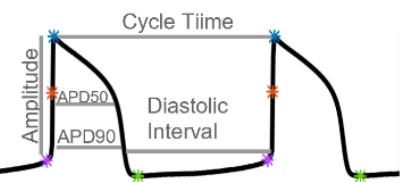

G
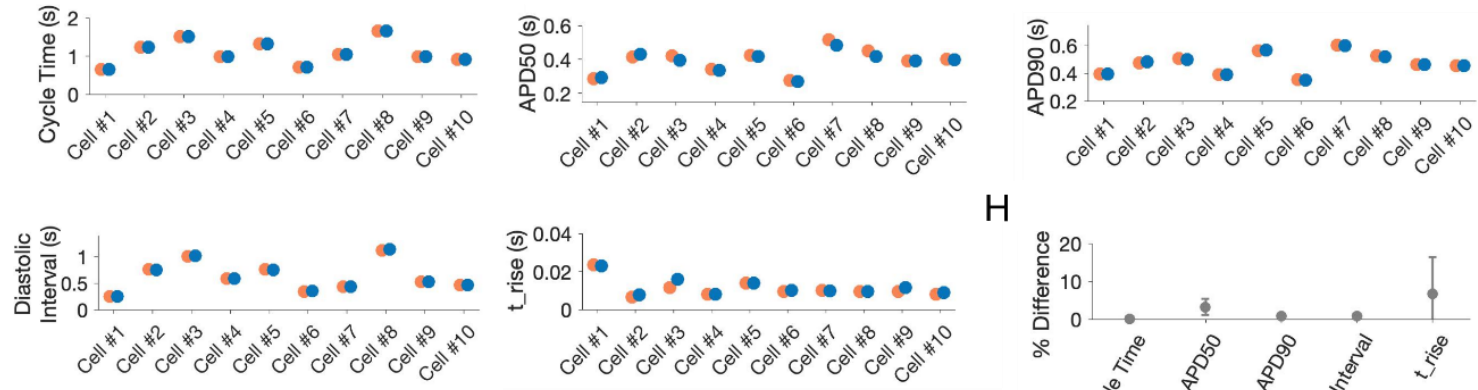

$\mathrm{H}$

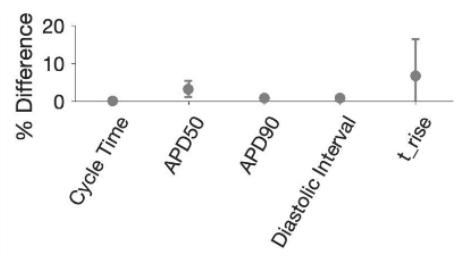

Figure 3. Simultaneous Patch clamp and NEA recordings verify the accuracy of NEA-recorded

iAPs. A) A schematic representation of simultaneous patch and NEA recording from the same cell. B) A brightfield image showing a patch pipette approaching a cell on the nanopillar electrode from the left. $C$ ) Simultaneous recording traces by patch clamp (orange) and NEA (blue) from the same cell. The two traces are aligned in time with $\mathrm{t}=0$ for electroporation. D) Zoomed-in time windows of NEA- and Patchrecordings before electroporation (left) and after electroporation (right). Before electroporation, NEA measured eAP spikes while patch clamp measured iAPs with high amplitudes. After electroporation, both NEA and patch measured iAPs. E) Three pairs of NEA- and Patch-recorded iAPs are amplitude normalized and overlaid together. The overlays show identical waveforms. F) Analyzed features of iAP waveforms: APD50, APD90, cycle time and diastolic interval and amplitudes. G) Analyzed values of iAP features for NEA- and patch-recorded iAPs for ten independent measurements. $H$ ) Averaged values of deviations of iAP features between NEAs and Patch for $\mathrm{N}=10$ cells. 


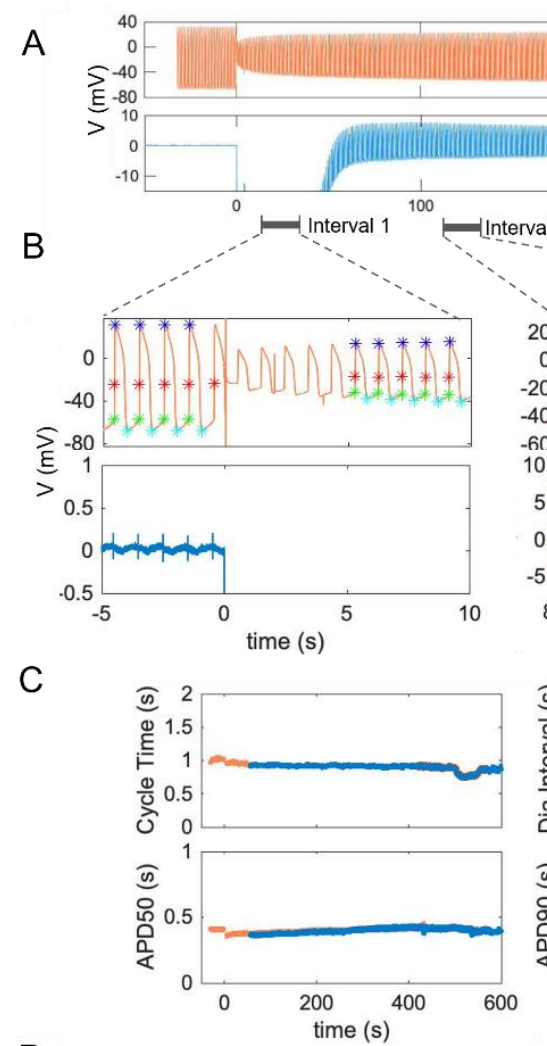

D
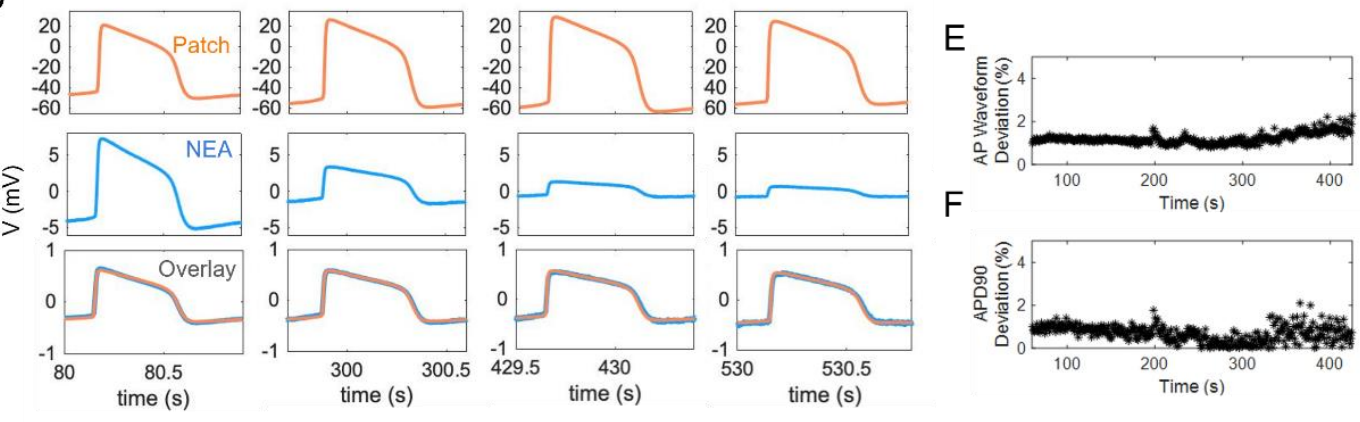

Figure 4. Time-dependent evolution of iAP features in simultaneous Patch clamp and NEA recordings. A) Simultaneous recording traces by patch clamp (orange) and NEA (blue) from the same cell. The two traces are aligned in time with $\mathrm{t}=0$ for electroporation. D) Zoomed-in time windows of NEAand Patch- recordings directly before and after electroporation (Interval 1), 80s after electroporation (Interval 2), and 425s after electroporation (Interval 3). The significant points on each iAP include spiking (red stars), starting (green stars), maximum (blue stars), and the minimum (cyan stars) points, which are determined automatically by a software. C) Overlay of time-dependent changes in APD features calculated for the Patch (Orange) or NEA (blue) recordings including cycle time (s), APD50 (s), APD90 (s), diastolic interval (s), amplitude $(\mathrm{mV})$ and spiking velocity- $\mathrm{dV} / \mathrm{dt}(\mathrm{mV} / \mathrm{s})$. D) Overlay of iAPs between patch (top-orange) and NEA (middle-blue) recordings at four time intervals after electroporation (80s, 300 s, 430s and 530s). As the membrane pores sealed over time, the amplitude of NEA-recorded iAPs decreased. Nevertheless, the scaled NEA and Patch recordings (Bottom row) largely overlap at all time points. E) Time dependent deviations of APD90 calculated from patch or NEA recordings. F) Time dependent deviations of iAP waveforms between patch and NEA recordings. 

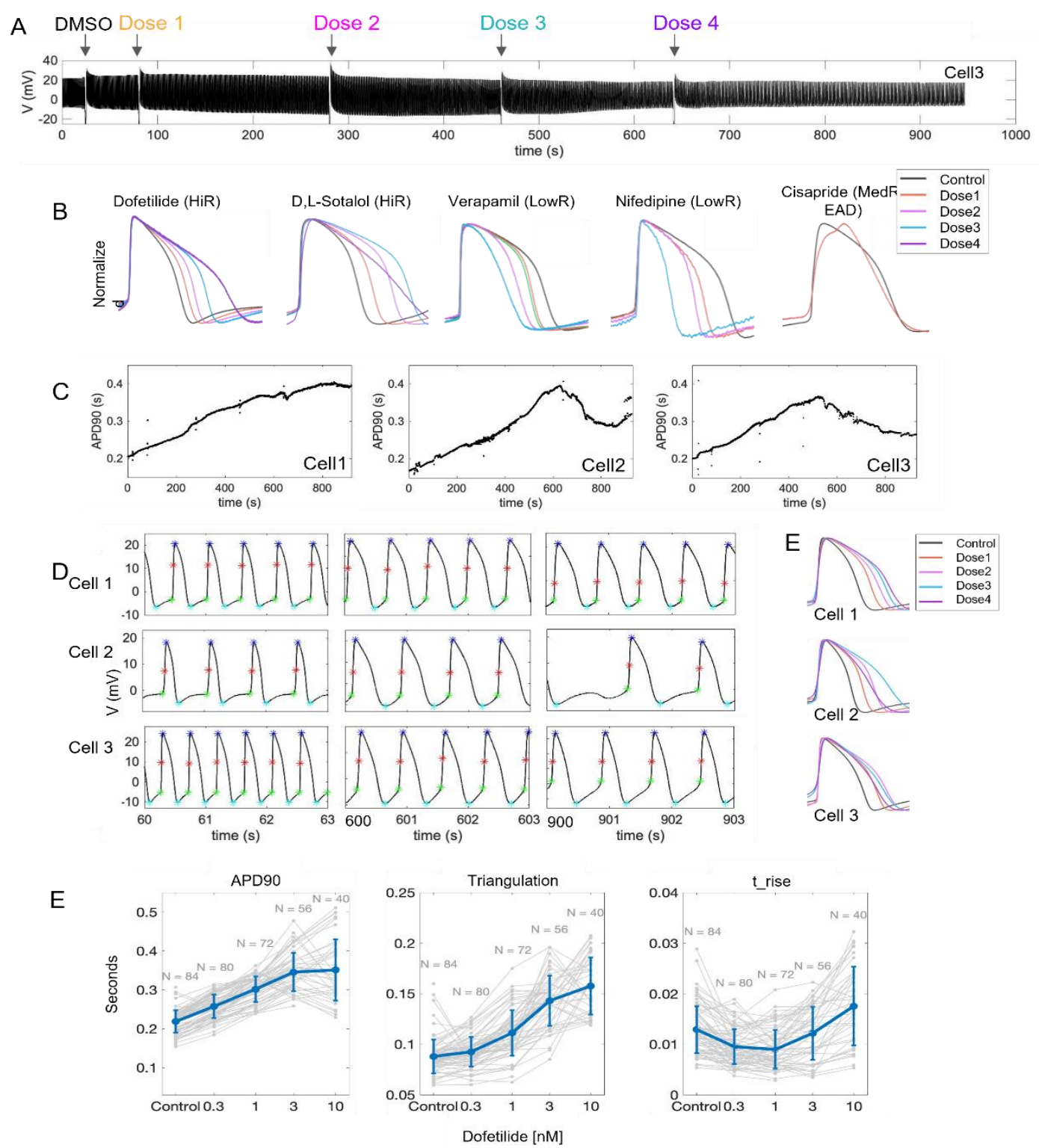

Figure 5. Self-referencing assessment of pharmacological compounds using nanocrown electrodes. A) A raw data trace of iAPs with sequential addition of pharmacological compounds (Dofetilide for the displayed trace). The large variations in the signal trace are due to vibrations associated with exchanging lipids when new doses of the drug are added (indicated by arrows). B) Overlay of amplitude-normalized and self-referencing iAPs, i.e. the same cell before and after adding increasing doses of each drug. The high risk drugs Dofetilide and D, L-Sotalol caused dose-dependent prolongation of iAPs, while the low-risk drugs Verapamil and Nifedipine caused dose-dependent shortening of iAPs. The medium-risk drug Cisapride caused early after-depolarization (EAD). C) Dose dependent changes in APD 90 for three cells over entire recording period showing distinct responses to Dofetilide. D) iAP waveforms of cells shown in part $C$, at various time points during the pharmacology experiments at 60s (DMSO), 600s (Dose3), and 900s (Dose4). At the highest dose, cell 2 missed an action potential at $900 \mathrm{~s}$. E) Self-referencing iAPs alignments for cells 1-3 show different dose-dependent responses to Dofetilide at doses 3 and 4. E) Self-referencing iAPs from 40-84 cells shows dosedependent changes in APD90, APD90-30 (triangulation), and rising time in response to Dofetilide. 
A
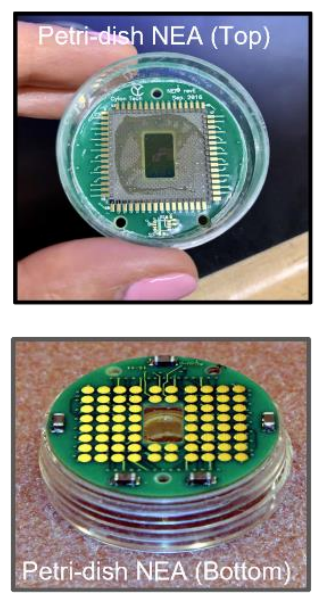

B

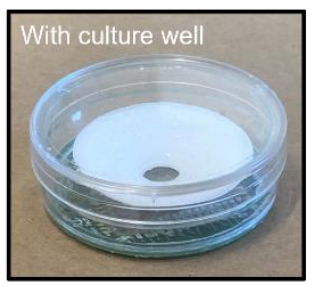

C

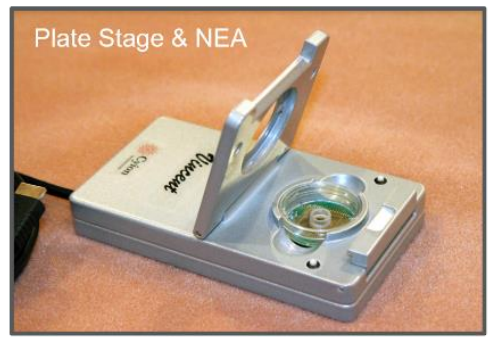

D

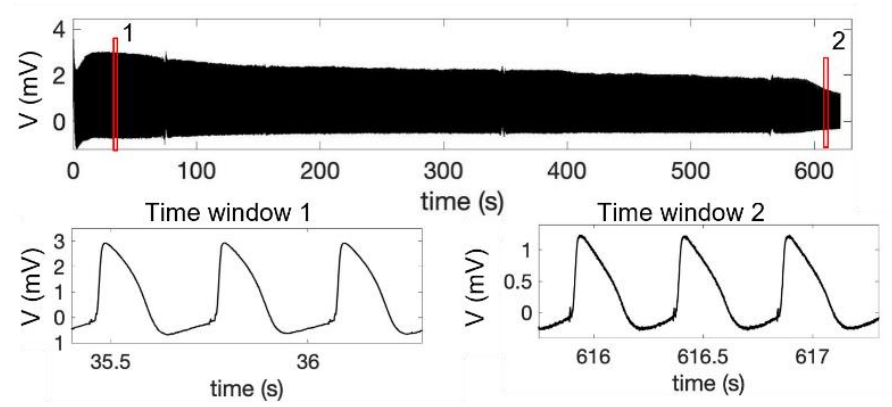

$\mathrm{E}$

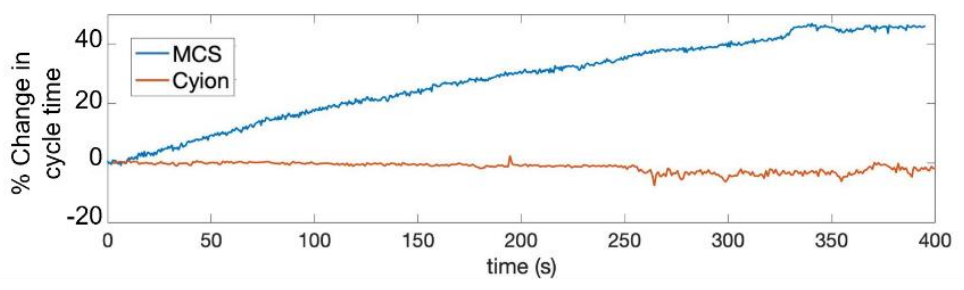

$\mathrm{F}$

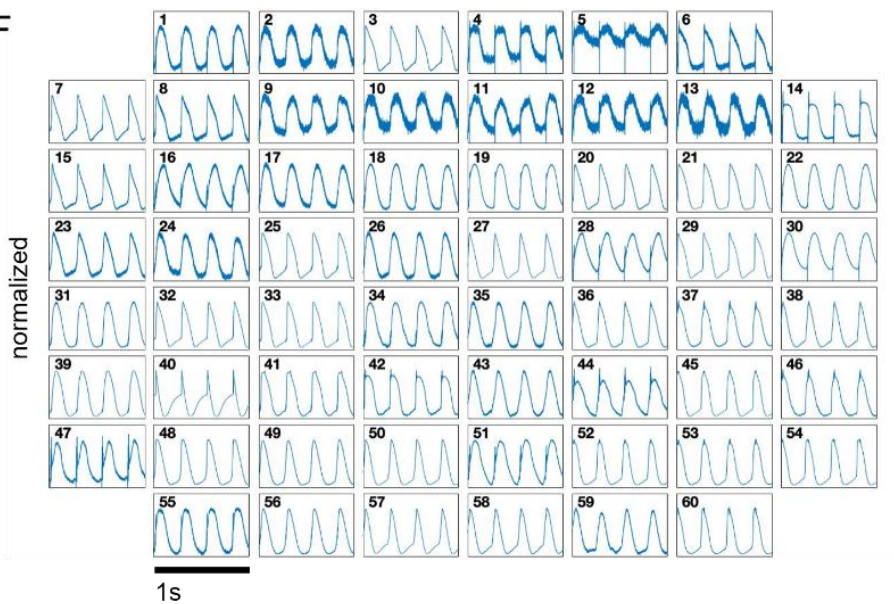

Figure 6. In-situ iAP recording using miniaturized petri-dish-like recording stage. A) Photographs of a Nanocrown device bound to a printed circuit board and integrated with a bottomless 35-mm petri dish. When placed on the recording stage, the electrode pads at the bottom are in contact to pogo pins inside the stage. B) A plastic 3D printed well (white) is assembled on top of the petri-dish NEA for cell culture. C) The petri dish NEA is situated in a Cyion recording stage. The recording stage is housed inside a $\mathrm{CO}_{2}$ incubator for in-situ recording. D) A raw data trace of in-situ recorded iAP using Cyion stage. R) Sequential electroporation allows the majority of channels to record iAPs. E) The measured cycle time of beating hiPSC-CMs gradually increases over 400s on the MCS recording stage (blue trace, outside the incubator), while the measured cycle time remains stable when using the miniaturized Cyion stage (orange trace, inside the incubator). F) iAPs recorded in parallel in a 60 channel NEA on the cyion system using sequential electroporation (A moving average filter with a window size of $1 \mathrm{~ms}$ was applied in $D$ and $\mathrm{F}$ to smooth signals).

\section{Supplementary Figures are included at the end}




\section{References}

Abbott, Jeffrey, Tianyang Ye, Donhee Ham, and Hongkun Park. 2018. "Optimizing

Nanoelectrode Arrays for Scalable Intracellular Electrophysiology." Accounts of Chemical Research 51 (3): 600-608.

Abbott, Jeffrey, Tianyang Ye, Keith Krenek, Rona S. Gertner, Steven Ban, Youbin Kim, Ling Qin, Wenxuan Wu, Hongkun Park, and Donhee Ham. 2020. "A Nanoelectrode Array for Obtaining Intracellular Recordings from Thousands of Connected Neurons." Nature Biomedical Engineering 4 (2): 232-41.

Abbott, Jeffrey, Tianyang Ye, Ling Qin, Marsela Jorgolli, Rona S. Gertner, Donhee Ham, and Hongkun Park. 2017. "CMOS Nanoelectrode Array for All-Electrical Intracellular Electrophysiological Imaging." Nature Nanotechnology 12 (5): 460-66.

Angle, Matthew R., Bianxiao Cui, and Nicholas A. Melosh. 2015. "Nanotechnology and Neurophysiology." Current Opinion in Neurobiology 32 (June): 132-40.

Bayer, R., R. Rodenkirchen, R. Kaufmann, J. H. Lee, and R. Hennekes. 1977. "The Effects of Nifedipine on Contraction and Monophasic Action Potential of Isolated Cat Myocardium." Naunyn-Schmiedeberg's Archives of Pharmacology 301 (1): 29-37.

Broyles, Connor, Paul Robinson, and Matthew Daniels. 2018. "Fluorescent, Bioluminescent, and Optogenetic Approaches to Study Excitable Physiology in the Single Cardiomyocyte." Cells. https://doi.org/10.3390/cells7060051.

Burridge, Paul W., Elena Matsa, Praveen Shukla, Ziliang C. Lin, Jared M. Churko, Antje D. Ebert, Feng Lan, et al. 2014. "Chemically Defined Generation of Human Cardiomyocytes." Nature Methods 11 (8): 855-60.

Cedrini, L., and G. Alloatti. 1979. "Effect of Temperature on the Electrical and Mechanical Activity of Lizard Ventricular Myocardium." Bollettino Della Societa Italiana Di Biologia Sperimentale 55 (14): 1362-68.

Colatsky, Thomas, Bernard Fermini, Gary Gintant, Jennifer B. Pierson, Philip Sager, Yuko Sekino, David G. Strauss, and Norman Stockbridge. 2016. "The Comprehensive in Vitro Proarrhythmia Assay (CiPA) Initiative - Update on Progress." Journal of Pharmacological and Toxicological Methods 81 (September): 15-20.

Crumb, William J., Jr, Jose Vicente, Lars Johannesen, and David G. Strauss. 2016. “An Evaluation of 30 Clinical Drugs against the Comprehensive in Vitro Proarrhythmia Assay (CiPA) Proposed lon Channel Panel." Journal of Pharmacological and Toxicological Methods 81 (September): 251-62.

Dana, Hod, Yi Sun, Boaz Mohar, Brad K. Hulse, Aaron M. Kerlin, Jeremy P. Hasseman, Getahun Tsegaye, et al. 2019. "High-Performance Calcium Sensors for Imaging Activity in Neuronal Populations and Microcompartments." Nature Methods 16 (7): 649-57.

Desbiolles, Benoît X. E., Etienne de Coulon, Nicolas Maïno, Arnaud Bertsch, Stephan Rohr, and Philippe Renaud. 2020. "Nanovolcano Microelectrode Arrays: Toward Long-Term onDemand Registration of Transmembrane Action Potentials by Controlled Electroporation." Microsystems \& Nanoengineering 6 (1): 67.

Desbiolles, B. X. E., E. de Coulon, A. Bertsch, S. Rohr, and P. Renaud. 2019. "Intracellular Recording of Cardiomyocyte Action Potentials with Nanopatterned Volcano-Shaped Microelectrode Arrays." Nano Letters 19 (9): 6173-81.

Dipalo, Michele, Hayder Amin, Laura Lovato, Fabio Moia, Valeria Caprettini, Gabriele C. Messina, Francesco Tantussi, Luca Berdondini, and Francesco De Angelis. 2017. "Intracellular and Extracellular Recording of Spontaneous Action Potentials in Mammalian Neurons and Cardiac Cells with 3D Plasmonic Nanoelectrodes." Nano Letters 17 (6): 
3932-39.

Dipalo, Michele, Giovanni Melle, Laura Lovato, Andrea Jacassi, Francesca Santoro, Valeria Caprettini, Andrea Schirato, et al. 2018. "Author Correction: Plasmonic Meta-Electrodes Allow Intracellular Recordings at Network Level on High-Density CMOS-Multi-Electrode Arrays." Nature Nanotechnology 13 (10): 972.

Dipalo, Michele, Sahil K. Rastogi, Laura Matino, Raghav Garg, Jacqueline Bliley, Giuseppina lachetta, Giovanni Melle, et al. 2021. "Intracellular Action Potential Recordings from Cardiomyocytes by Ultrafast Pulsed Laser Irradiation of Fuzzy Graphene Microelectrodes." Science Advances 7 (15). https://doi.org/10.1126/sciadv.abd5175.

Duan, Xiaojie, Ruixuan Gao, Ping Xie, Tzahi Cohen-Karni, Quan Qing, Hwan Sung Choe, Bozhi Tian, Xiaocheng Jiang, and Charles M. Lieber. 2011. "Intracellular Recordings of Action Potentials by an Extracellular Nanoscale Field-Effect Transistor." Nature Nanotechnology 7 (3): 174-79.

Du, David T. M., Nicola Hellen, Christopher Kane, and Cesare M. N. Terracciano. 2015. "Action Potential Morphology of Human Induced Pluripotent Stem Cell-Derived Cardiomyocytes Does Not Predict Cardiac Chamber Specificity and Is Dependent on Cell Density." Biophysical Journal 108 (1): 1-4.

Dunlop, John, Mark Bowlby, Ravikumar Peri, Dmytro Vasilyev, and Robert Arias. 2008. "HighThroughput Electrophysiology: An Emerging Paradigm for lon-Channel Screening and Physiology." Nature Reviews. Drug Discovery 7 (4): 358-68.

Edwards, Stacie L., Viviana Zlochiver, Donald B. Conrad, Ravi Vaidyanathan, Andrew M. Valiquette, and Rosy Joshi-Mukherjee. 2018. "A Multiwell Cardiac $\mu$ GMEA Platform for Action Potential Recordings from Human iPSC-Derived Cardiomyocyte Constructs." Stem Cell Reports 11 (2): 522-36.

Gibson, John K., Yimei Yue, Jared Bronson, Cassie Palmer, and Randy Numann. 2014. "Human Stem Cell-Derived Cardiomyocytes Detect Drug-Mediated Changes in Action Potentials and Ion Currents." Journal of Pharmacological and Toxicological Methods 70 (3): 255-67.

Hai, Aviad, Joseph Shappir, and Micha E. Spira. 2010. "In-Cell Recordings by Extracellular Microelectrodes." Nature Methods 7 (3): 200-202.

Hayes, Heather B., Anthony M. Nicolini, Colin A. Arrowood, Stacie A. Chvatal, David W. Wolfson, Hee Cheol Cho, Denise D. Sullivan, et al. 2019. "Novel Method for Action Potential Measurements from Intact Cardiac Monolayers with Multiwell Microelectrode Array Technology." Scientific Reports 9 (1): 11893.

Hondeghem, Luc M., and Peter Hoffmann. 2003. "Blinded Test in Isolated Female Rabbit Heart Reliably Identifies Action Potential Duration Prolongation and Proarrhythmic Drugs: Importance of Triangulation, Reverse Use Dependence, and Instability." Journal of Cardiovascular Pharmacology 41 (1): 14-24.

Hyun, Soo-Wang, Bo-Ram Kim, Sung-Ae Hyun, and Joung-Wook Seo. 2017. "The Assessment of Electrophysiological Activity in Human-Induced Pluripotent Stem Cell-Derived Cardiomyocytes Exposed to Dimethyl Sulfoxide and Ethanol by Manual Patch Clamp and Multi-Electrode Array System." Journal of Pharmacological and Toxicological Methods 87 (September): 93-98.

Kimura, S., A. L. Bassett, H. Xi, and R. J. Myerburg. 1992. "Verapamil Diminishes Action Potential Changes during Metabolic Inhibition by Blocking ATP-Regulated Potassium Currents." Circulation Research 71 (1): 87-95.

Kohlhardt, M., and K. Haap. 1976. "The Response of Ca-Mediated Action Potentials and Contractile Activity in Mammalian Ventricular Myocardium towards Alkalosis." Experientia $32(2): 150-52$.

Kramer, James, Herbert M. Himmel, Anders Lindqvist, Sonja Stoelzle-Feix, Khuram W. Chaudhary, Dingzhou Li, Georg Andrees Bohme, et al. 2020. "Publisher Correction: Cross- 
Site and Cross-Platform Variability of Automated Patch Clamp Assessments of Drug Effects on Human Cardiac Currents in Recombinant Cells." Scientific Reports 10 (1): 11884.

Liang, Ping, Feng Lan, Andrew S. Lee, Tingyu Gong, Veronica Sanchez-Freire, Yongming Wang, Sebastian Diecke, et al. 2013. "Drug Screening Using a Library of Human Induced Pluripotent Stem Cell-Derived Cardiomyocytes Reveals Disease-Specific Patterns of Cardiotoxicity." Circulation. https://doi.org/10.1161/circulationaha.113.001883.

Lin, Ziliang Carter, Allister F. McGuire, Paul W. Burridge, Elena Matsa, Hsin-Ya Lou, Joseph C. Wu, and Bianxiao Cui. 2017. "Accurate Nanoelectrode Recording of Human Pluripotent Stem Cell-Derived Cardiomyocytes for Assaying Drugs and Modeling Disease." Microsystems \& Nanoengineering 3 (March): 16080.

Lin, Ziliang Carter, Chong Xie, Yasuko Osakada, Yi Cui, and Bianxiao Cui. 2014. "Iridium Oxide Nanotube Electrodes for Sensitive and Prolonged Intracellular Measurement of Action Potentials." Nature Communications. https://doi.org/10.1038/ncomms4206.

Liu, Ren, Renjie Chen, Ahmed T. Elthakeb, Sang Heon Lee, Sandy Hinckley, Massoud L. Khraiche, John Scott, et al. 2017. "High Density Individually Addressable Nanowire Arrays Record Intracellular Activity from Primary Rodent and Human Stem Cell Derived Neurons." Nano Letters 17 (5): 2757-64.

Li, Wener, Xiaojing Luo, Ying Ulbricht, Michael Wagner, Christopher Piorkowski, Ali ElArmouche, and Kaomei Guan. 2019. "Establishment of an Automated Patch-Clamp Platform for Electrophysiological and Pharmacological Evaluation of hiPSC-CMs." Stem Cell Research 41 (December): 101662.

Lopez, Carolina Mora, Ho Sung Chun, Shiwei Wang, Laurent Berti, Jan Putzeys, Carl Van Den Bulcke, Jan-Willem Weijers, et al. 2018. "A Multimodal CMOS MEA for High-Throughput Intracellular Action Potential Measurements and Impedance Spectroscopy in DrugScreening Applications." IEEE Journal of Solid-State Circuits. https://doi.org/10.1109/jssc.2018.2863952.

Lou, Hsin-Ya, Wenting Zhao, Xiao Li, Liting Duan, Alexander Powers, Matthew Akamatsu, Francesca Santoro, et al. 2019. "Membrane Curvature Underlies Actin Reorganization in Response to Nanoscale Surface Topography." Proceedings of the National Academy of Sciences of the United States of America 116 (46): 23143-51.

Marschang, H., T. Beyer, L. Karolyi, W. Kübler, and J. Brachmann. 1998. "Differential Rate and Potassium-Dependent Effects of the Class III Agents D-Sotalol and Dofetilide on Guinea Pig Papillary Muscle." Cardiovascular Drugs and Therapy / Sponsored by the International Society of Cardiovascular Pharmacotherapy 12 (6): 573-83.

McGuire, Allister F., Francesca Santoro, and Bianxiao Cui. 2018. "Interfacing Cells with Vertical Nanoscale Devices: Applications and Characterization." Annual Review of Analytical Chemistry 11 (1): 101-26.

Robinson, Jacob T., Marsela Jorgolli, Alex K. Shalek, Myung-Han Yoon, Rona S. Gertner, and Hongkun Park. 2012. "Vertical Nanowire Electrode Arrays as a Scalable Platform for Intracellular Interfacing to Neuronal Circuits." Nature Nanotechnology 7 (3): 180-84.

Scheel, Olaf, Stefanie Frech, Bogdan Amuzescu, Jörg Eisfeld, Kun-Han Lin, and Thomas Knott. 2014. "Action Potential Characterization of Human Induced Pluripotent Stem Cell-Derived Cardiomyocytes Using Automated Patch-Clamp Technology." ASSAY and Drug Development Technologies. https://doi.org/10.1089/adt.2014.601.

Shroff, Sanaya N., Shoshana L. Das, Hua-An Tseng, Jad Noueihed, Fernando Fernandez, John A. White, Christopher S. Chen, and Xue Han. 2020. "Voltage Imaging of Cardiac Cells and Tissue Using the Genetically Encoded Voltage Sensor Archon1." iScience 23 (4): 100974.

Spira, Micha E., and Aviad Hai. 2013. "Multi-Electrode Array Technologies for Neuroscience and Cardiology." Nature Nanotechnology 8 (2): 83-94.

Stoelzle, Sonja, Alison Haythornthwaite, Ralf Kettenhofen, Eugen Kolossov, Heribert Bohlen, 
Michael George, Andrea Brüggemann, and Niels Fertig. 2011. "Automated Patch Clamp on mESC-Derived Cardiomyocytes for Cardiotoxicity Prediction." Journal of Biomolecular Screening 16 (8): 910-16.

Strauss, David G., Gary Gintant, Zhihua Li, Wendy Wu, Ksenia Blinova, Jose Vicente, J. Rick Turner, and Philip T. Sager. 2019. "Comprehensive In Vitro Proarrhythmia Assay (CiPA) Update from a Cardiac Safety Research Consortium / Health and Environmental Sciences Institute / FDA Meeting." Therapeutic Innovation \& Regulatory Science. https://doi.org/10.1177/2168479018795117.

Wu, Haodi, Huaxiao Yang, June-Wha Rhee, Joe Z. Zhang, Chi Keung Lam, Karim Sallam, Alex C. Y. Chang, et al. 2019. "Modelling Diastolic Dysfunction in Induced Pluripotent Stem CellDerived Cardiomyocytes from Hypertrophic Cardiomyopathy Patients." European Heart Journal 40 (45): 3685-95.

Xie, Chong, Ziliang Lin, Lindsey Hanson, Yi Cui, and Bianxiao Cui. 2012. "Intracellular Recording of Action Potentials by Nanopillar Electroporation." Nature Nanotechnology 7 (3): 185-90.

Ye, Shiqiao, Xiaoping Wan, Juan Su, Akshar Patel, Blake Justis, Isabelle Deschênes, and MingTao Zhao. 2021. "Generation and Expansion of Human Cardiomyocytes from Patient Peripheral Blood Mononuclear Cells." Journal of Visualized Experiments: JoVE, no. 168 (February). https://doi.org/10.3791/62206.

Zhao, Ming-Tao, Haodong Chen, Qing Liu, Ning-Yi Shao, Nazish Sayed, Hung-Ta Wo, Joe Z. Zhang, et al. 2017. "Molecular and Functional Resemblance of Differentiated Cells Derived from Isogenic Human iPSCs and SCNT-Derived ESCs." Proceedings of the National Academy of Sciences of the United States of America 114 (52): E11111-20.

Zhao, Wenting, Lindsey Hanson, Hsin-Ya Lou, Matthew Akamatsu, Praveen D. Chowdary, Francesca Santoro, Jessica R. Marks, et al. 2017. "Nanoscale Manipulation of Membrane Curvature for Probing Endocytosis in Live Cells." Nature Nanotechnology 12 (8): 750-56.

Zhu, Renjun, Michal A. Millrod, Elias T. Zambidis, and Leslie Tung. 2016. "Variability of Action Potentials Within and Among Cardiac Cell Clusters Derived from Human Embryonic Stem Cells." Scientific Reports 6 (January): 18544. 